\title{
Sigmatropic Rearrangements as Tools for Amino Acid and Peptide Modification: Application of the Allylic Sulfur Ylide Rearrangement to the Preparation of Neoglyco- and Other Conjugates
}

\author{
David Crich ${ }^{*}$, Yekui Zou, and Franck Brebion \\ Department of Chemistry, University of Illinois at Chicago, 845 West Taylor Street, Chicago, Illinois \\ 60607-7061
}

\begin{abstract}
Reaction of $S$-allyl cysteine derivatives, generated by the selenocysteine ligation, with rhodium carbenoids, stabilized and unstabilized, enables the attachment of diverse functionality onto cysteine residues. The reaction is successfully applied to the introduction of lipid-like residues, a fluorous alkyl chain, and mono and disaccharides.
\end{abstract}

\section{Introduction}

Recently, we have described methods for the permanent ligation of thiols involving the coupling of either $S e$-allyl Bunte salts ( $S e$-allyl selenosulfonates), or $S$-allyl $S^{\prime}$-heteroaryl disulfides, with thiols to give $S e$-allyl selenosulfides or $S$-allyl disulfides, respectively, followed by a dechalcogenative 2,3-sigmatropic rearrangement to give the ligated products (Scheme 1). ${ }^{1}$ These complementary reactions, for which all steps take place at room temperature in protic media, were illustrated by the introduction of a range of allyl and prenyl groups to cysteine and other thiols.

By virtue of the reaction mechanism these reactions afford allylic sulfides as products, thereby opening up avenues for further functionalization, one of which is the 2,3-sigmatropic rearrangement of allylic sulfur ylides as we describe here.

The 2,3-sigmatropic rearrangement of allylic sulfur ylides has been known for many years and has found widespread application in organic synthesis. ${ }^{2}$ With an eye to eventual applications in the modification of peptides, proteins and other bioconjugates, for our investigation we selected the modification of this reaction popularized by Kirmse and Doyle, in which the sulfur ylide is generated by transition metal catalyzed addition of a diazoalkane to an allylic sulfide (Scheme 2). ${ }^{3}$ Our choice of the Kirmse-Doyle reaction was further guided by current interest in the deployment of transition metal-catalyzed reactions in peptide chemistry, ${ }^{4}$ and more particularly by the recent publication of Francis on the reaction of a stabilized vinyl diazo acetate, catalyzed by dirhodium tetracetate, with tryptophan residues in horse heart myoglobin and substilin Carlsberg in aqueous ethylene glycol. ${ }^{5}$ 


\section{Results and Discussion}

We began with a feasibility study in which a series of diazoalkanes were allowed to react with $S$-allyl or $S$-methylallyl cysteine derivatives, obtained by the selenosulfide ligation method, in the presence of catalytic $\mathrm{Rh}_{2}(\mathrm{OAc})_{4}$ in dimethoxyethane at room temperature. From the results of these experiments (Table 1) it is clear that a variety of simple alkyl groups may be introduced into cysteine in this manner, with moderate yields consistent with earlier studies on simple allylic sulfides. ${ }^{3 a-d, 6}$ In each case the new stereogenic center formed as a result of the sigmatropic rearrangement was obtained as an approximately 1:1 mixture of isomers.

Attention was turned to the functionalization of a tripeptide 7 (Table 2). Given the importance of the introduction of lipids onto cysteine in peptide and protein chemistry and biochemistry, 7 entries 3 and 4 of Table 2 are especially noteworthy. In view of the recent interest in the fluorous tagging of peptides and proteins, attention is also called to entry 5 of Table 2.8

With the exception of the tryptophan case discussed below, the main byproducts from the chemistry presented here are those of dimerization of the intermediate metal carbenoids, as is typical of this type of reaction. Analysis of crude reaction mixtures by NMR spectroscopy indicates that the mass balance of the amino acid or peptide derivatives is made up largely by the unreacted substrate; insertion into the peptide or carbamate $\mathrm{NH}$ bond is not a major problem, as anticipated from the work of Francis. ${ }^{5}$

Interest in the glycosylation of cysteine residues as a means of peptide and protein glycosylation ${ }^{7 \mathrm{a}, 9}$ led us to investigate carbohydrate-based diazoalkanes. To this end, peracetyl $\beta$ - ${ }^{-}$-glucosyl and $\beta$ - ${ }^{-}$-chitobiosyl diazo amides $\mathbf{1 3}$ and $\mathbf{1 4}$ were obtained from the glycosyl amines, via the tosyl hydrazones. An important feature in the design of $\mathbf{1 3}$ and $\mathbf{1 4}$ was the use of the diazoamide function rather than the much more common diazoesters. This choice was made based on the trans-nature of the amide bond, with its high barrier to inversion relative to the ester bond, which it was anticipated would prevent the metal carbenoid intermediate from "biting back" on the carbohydrate moiety. This supposition was borne out in practice, as the only carbohydrate-based byproducts observed upon activation with $\mathrm{Rh}_{2}(\mathrm{OAc})_{4}$ were those resulting from dimerization of the carbenoid, which is typical for this type of reaction.

Glucosyl diazoamide 13 was attached to allyl hexadecyl sulfide (15) to establish the validity of the method (Table 3 ) before couplings to amino acid and peptide-based sulfides were undertaken (Table 3, entries 2-5), providing access to a new class of neoglycoconjugates. ${ }^{10}$ It is especially noteworthy that, although glycoamino acids and peptides 16-20 are cysteine derivatives, the amide linkage employed opens up the possibility of the application of this chemistry, coupled with native peptide ligation ${ }^{11}$ and our dechalcogenative allylation protocols, ${ }^{1}$ as mimics of the $N$-linked glycoproteins, ${ }^{12}$ for which new methods are constantly being sought. $9 \mathrm{~d}, \mathrm{e}, 13$

Finally, in view of the work of Francis, ${ }^{5}$ we briefly investigated chemoselectivity with $S$ methallyl Boc-L-Cys-L-Ala-L-Trp-OMe ${ }^{1}$ with a diazoketone. Literature work on the addition of $\mathrm{Rh}$ carbenoids to sulfides in the presence of indoles provided grounds for optimism that our chemistry would be applicable in the presence of tryptophan, ${ }^{14}$ however, complex reaction mixtures were obtained from which only two products, $\mathbf{2 1}$ and $\mathbf{2 2}$, were obtained pure in low yield (18 and 9\%, respectively). The insertion of stabilized rhodium carbenoids into the indole $\mathrm{N}-\mathrm{H}$ bond, as in the formation of $\mathbf{2 2}$, is a known reaction pathway, ${ }^{15}$ and is consistent with the structures proposed by Francis for reaction with protein-based tryptophan residues. ${ }^{5}$ At least for the present, it appears that the application of the Doyle-Kirmse reaction to $S$-allylated peptides and proteins is not compatible with the presence of tryptophan. ${ }^{16}$ 

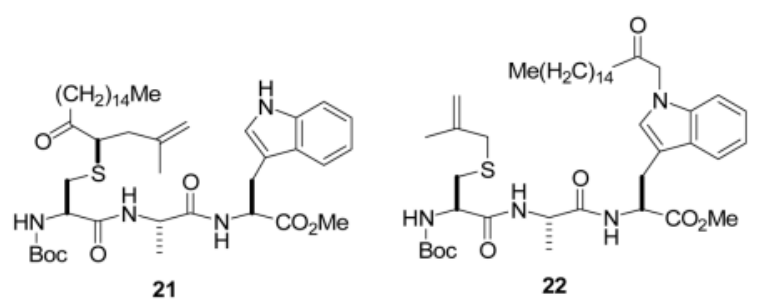

\section{Experimental Section}

Phenyl diazomethane was prepared following the procedure reported by Creary. ${ }^{17}$

1-Diazo-2-heptadecanone was prepared following the procedure reported by Scott and Sumpter. ${ }^{18}$ A freshly prepared solution of diazomethane $(30 \mathrm{mmol})$ in anhydrous ether $(60$ $\mathrm{mL}$ ) was cooled to $0^{\circ} \mathrm{C}$ and stirred at high speed. To this cooled solution, hexadecanoyl chloride $(2.75 \mathrm{~g}, 10 \mathrm{mmol})$ in anhydrous ether $(20 \mathrm{~mL})$ was added dropwise over $20 \mathrm{~min}$. The resulting reaction mixture was stirred cold for an additional $30 \mathrm{~min}$ and then at room temperature for 60 $\mathrm{min}$. After this period of time the reaction was complete, and excess diazomethane was removed by evacuating the flask with a water aspirator pump in the hood. After the diazomethane has been removed, the remaining ethereal solution was concentrated by rotary evaporation to give crude compound. Pure 1-diazo-2-heptadecanone was obtained as yellow solid ( $2.61 \mathrm{~g}, 93 \%$ yield) by chromatography on silica gel using $10 \%$ ethyl acetate/hexane as an eluent. ${ }^{1} \mathrm{H}$ NMR: $\delta 5.24(\mathrm{~s}, 1 \mathrm{H}), 2.27-2.38(\mathrm{~m}, 2 \mathrm{H}), 1.58-1.64(\mathrm{~m}, 2 \mathrm{H}), 1.23$ (br. s, 24H), $0.86(\mathrm{t}, J=7.0$, 3H). ${ }^{13}$ C NMR: $\delta$ 195.4, 54.2, 41.1, 31.9, 29.7 (3C), 29.6 (2C), 29.5 (2C), 29.4 (2C), 29.2, 25.3, 22.7, 14.1. v 2120, 2100, $1620 \mathrm{~cm}^{-1}$. EIHRMS Calcd for $\mathrm{C}_{17} \mathrm{H}_{32} \mathrm{~N}_{2} \mathrm{O}[\mathrm{M}]^{+}: 280.2515$, found: 280.2520 .

\section{1-Diazoundecane}

1-Diazoundecane was prepared following the procedure reported by Shechter and Holton. ${ }^{19}$ Undecanal $(1.36 \mathrm{~g}, 8.0 \mathrm{mmol})$ was added to stirred anhydrous hydrazine $(2.56 \mathrm{~g}, 80.0 \mathrm{mmol})$ at $55^{\circ} \mathrm{C}$. The reaction was continued $45 \mathrm{~min}$ at $55-65^{\circ} \mathrm{C}$. After the mixture had been cooled to room temperature, methylene chloride $(25 \mathrm{~mL})$ was added. The solution was washed with saturated aqueous sodium chloride $(3 \times 10 \mathrm{~mL})$, dried over potassium carbonate, and concentrated under reduced pressure to a volume of $5 \mathrm{~mL}$. Dimethylformamide $(10 \mathrm{~mL})$ was added, and remaining methylene chloride was removed by vacuum evaporation. The solution of undecanal hydrazone in dimethylformamide was cooled to $-78^{\circ} \mathrm{C}(15 \mathrm{~min})$ and diluted with cold tetramethylguanidine $(4 \mathrm{~mL})$. Lead tetraacetate $(3.90 \mathrm{~g}, 8.8 \mathrm{mmol})$ was added in $5 \mathrm{~min}$, and the mixture was stirred $60 \mathrm{~min}$ at $-78{ }^{\circ} \mathrm{C}$. The reaction solution was diluted with cold hexane $(3 \times 20 \mathrm{~mL})$ and extracted at $-78^{\circ} \mathrm{C}$. The combined cold hexane extracts were washed with cold $\left(-30^{\circ} \mathrm{C}\right) 30 \%$ aqueous potassium hydroxide $(2 \times 10 \mathrm{~mL})$, small pieces of dry ice were added, and the solution was then filtered to give a rose-red solution of 1-diazoundecane in hexane which was used directly in further reaction.

\section{9-Diazo-1,1,1,2,2,3,3,4,4,5,5,6,6-tridecafluorononane}

4,4,5,5,6,6,7,7,8,8,9,9,9-Tridecafluorononanal $(8.0 \mathrm{mmol})$ was added to stirred anhydrous hydrazine $(2.56 \mathrm{~g}, 80.0 \mathrm{mmol})$ at $55^{\circ} \mathrm{C}$. The reaction was continued $45 \mathrm{~min}$ at $55-65^{\circ} \mathrm{C}$. After the mixture had been cooled to room temperature, methylene chloride $(25 \mathrm{~mL})$ was added. The solution was washed with saturated aqueous sodium chloride $(3 \times 10 \mathrm{~mL})$, dried over potassium carbonate, and concentrated under reduced pressure to a volume of $5 \mathrm{~mL}$. Dimethylformamide $(10 \mathrm{~mL})$ was added, and remaining methylene chloride was removed by vacuum volatization. The solution of undecylic aldehyde hydrazone in dimethylformamide was cooled to $-78^{\circ} \mathrm{C}$ $(15 \mathrm{~min})$ and diluted with cold tetramethylguanidine $(4 \mathrm{~mL})$. Lead tetraacetate $(3.90 \mathrm{~g}, 8.8$ 
mmol) was added in $5 \mathrm{~min}$, and the mixture was stirred $60 \mathrm{~min}$ at $-78^{\circ} \mathrm{C}$. The reaction solution was diluted with cold hexane $(3 \times 20 \mathrm{~mL})$ and extracted at $-78^{\circ} \mathrm{C}$. The combined cold hexane extracts were washed with cold $\left(-30^{\circ} \mathrm{C}\right) 30 \%$ aqueous potassium hydroxide $(2 \times 10 \mathrm{~mL})$, small pieces of dry ice were added, and the solution was then filtered to give a rose-red solution of 1-diazo-undecane in hexane which was used directly.

\section{$N$-(2,3,4,6-Tetra-O-acetyl- $\beta$-D-glucopyranosyl)diazoacetamide}

To a solution of tetra- $O$-acetyl- $\beta$-D-glucopyranosylamine $(695 \mathrm{mg}, 2.0 \mathrm{mmol}, 1.0$ equiv.) and glyoxylic acid p-toluenesulfonylhydrazone $2017,18(533 \mathrm{mg}, 2.2 \mathrm{mmol}, 1.1$ equiv.) in ice-cold THF (20 mL) was added, dropwise, DCC (454 mg, $2.2 \mathrm{mmol}, 1.1$ equiv.) in THF (10 mL). The mixture was allowed to warm to room temperature and stirring was continued over night. Then the solid was filtered off. After removal of the solvent, the filtrate was purified by flash chromatography (hexanes/EtOAc, 2:3) to afford a yellow solid (582 $\mathrm{mg}$, 51\% yield)

To a solution of the yellow solid ( $100 \mathrm{mg}, 0.175 \mathrm{mmol}, 1.0$ equiv.) in methylene chloride (5.0 $\mathrm{mL})$ was added triethylamine $(0.35 \mathrm{mmol}, 2.0$ equiv. $)$ in a nitrogen atmosphere. The mixture was stirred at room temperature over night. Then the solution was diluted with ethyl acetate $(20 \mathrm{~mL})$ and washed by water $(2 \times 10 \mathrm{~mL})$, brine $(2 \times 10 \mathrm{~mL})$, dried over sodium sulfate and concentrated. The remaining residue was then purified by flash chromatography (hexanes/ EtOAc, 2:3) to afford the diazo compound ( $65 \mathrm{mg}$, 90\% yield) as a viscous yellow oil. ${ }^{1} \mathrm{H}$ NMR: $\delta 6.10(\mathrm{~d}, J=9.5 \mathrm{~Hz}, 1 \mathrm{H}), 5.26-5.31(\mathrm{~m}, 2 \mathrm{H}), 5.03(\mathrm{t}, J=9.5 \mathrm{~Hz}, 1 \mathrm{H}), 4.88(\mathrm{t}, J=9.5$, $1 \mathrm{H}), 4.81(\mathrm{~s}, 1 \mathrm{H}), 4.29(\mathrm{dd}, J=12.5,4.5 \mathrm{~Hz}, 1 \mathrm{H}), 4.06(\mathrm{dd}, J=12.5,2.0 \mathrm{~Hz}, 1 \mathrm{H}), 3.80-3.94$ (m, 1H), 2.00-2.06 (m, 12H). ${ }^{13} \mathrm{C}$ NMR: $\delta$ 171.2, 170.7, 169.9, 169.7, 165.9, 78.5, 73.4, 72.7, 70.5, 68.2, 61.7, 48.1, 20.7 (4C). v 2111, 1750, $1653 \mathrm{~cm}^{-1}$. ESIHRMS Calcd for $\mathrm{C}_{16} \mathrm{H}_{21} \mathrm{~N}_{3} \mathrm{O}_{10} \mathrm{Na}[\mathrm{M}+\mathrm{Na}]^{+}$: 438.1125, found: 438.1128 .

\section{$N-\left(2,3,6,2^{\prime}, 3^{\prime}, 4^{\prime}, 6^{\prime}\right.$-Hepta-O-acetyl- $\beta$-cellobiosyl)-diazoacetamide}

To a solution of hepta- $O$-acetyl- $\beta$-cellobiosylamine $(857 \mathrm{mg}, 1.35 \mathrm{mmol}, 1.0$ equiv.) and glyoxylic acid p-toluenesulfonylhydrazone $\mathrm{e}^{5,6}(360 \mathrm{mg}, 1.49 \mathrm{mmol}, 1.1$ equiv. $)$ in ice-cold THF (10 mL) was added, dropwise, DCC (306 mg, $1.49 \mathrm{mmol}, 1.1$ equiv.) in THF (10 mL). The mixture was allowed to warm to room temperature and stirring was continued over night. Then the solid was filtered off. After removal of the solvent, the filtrate was purified by flash chromatography (hexanes/EtOAc, 2:3) to afford a yellow solid (735 mg, 63\% yield)

To a solution of the yellow solid ( $739 \mathrm{mg}, 0.86 \mathrm{mmol}, 1.0$ equiv.) in methylene chloride (10 $\mathrm{mL}$ ) was added triethylamine ( $1.72 \mathrm{mmol}, 2.0$ equiv.) in a nitrogen atmosphere. The mixture was stirred at room temperature over night. Then the solution was diluted with ethyl acetate $(20 \mathrm{~mL})$ and washed by water $(2 \times 10 \mathrm{~mL})$, brine $(2 \times 10 \mathrm{~mL})$, dried over sodium sulfate and concentrated. The remaining residue was then purified by flash chromatography (hexanes/ EtOAc, 2:3) to afford the diazo compound (560 mg, 93\% yield) as a yellow solid. ${ }^{1} \mathrm{H}$ NMR: $\delta 5.98(\mathrm{~d}, J=9.5 \mathrm{~Hz}, 1 \mathrm{H}), 5.22-5.26(\mathrm{~m}, 2 \mathrm{H}), 5.12(\mathrm{t}, J=9.5 \mathrm{~Hz}, 1 \mathrm{H}), 5.05(\mathrm{t}, J=9.5 \mathrm{~Hz}, 1 \mathrm{H})$, $4.89(\mathrm{t}, J=9.5 \mathrm{~Hz}, 1 \mathrm{H}), 4.79(\mathrm{t}, J=9.5 \mathrm{~Hz}, 1 \mathrm{H}), 4.78(\mathrm{~s}, 1 \mathrm{H}), 4.48(\mathrm{dd}, J=12.5,4.5 \mathrm{~Hz}, 1 \mathrm{H})$, $4.42(\mathrm{dd}, J=12.5,4.5 \mathrm{~Hz}, 1 \mathrm{H}), 4.34(\mathrm{dd}, J=12.5,4.5 \mathrm{~Hz}, 1 \mathrm{H}), 4.07-4.12(\mathrm{~m}, 2 \mathrm{H}), 4.01(\mathrm{dd}$, $J=12.5,2.0 \mathrm{~Hz}, 1 \mathrm{H}), 3.72-3.76(\mathrm{~m}, 2 \mathrm{H}), 3.62-3.65(\mathrm{~m}, 1 \mathrm{H}), 1.95-2.12(\mathrm{~m}, 21 \mathrm{H}) .{ }^{13} \mathrm{C}$ NMR: $\delta$ 171.1, 170.5, 170.3, 170.2, 169.5, 169.3, 169.1, 166.1, 100.6, 78.3, 76.2, 74.5, 72.9, 72.5, 71.8, 71.5, 70.7, 67.8, 62.0, 61.5, 60.4, 47.9, 20.7. $v 2111,1751,1654 \mathrm{~cm}^{-1}$. ESIHRMS Calcd for $\mathrm{C}_{28} \mathrm{H}_{37} \mathrm{~N}_{3} \mathrm{O}_{18} \mathrm{Na}$ [M+Na]+: 726.1970, found: 726.1981 .

\section{General Procedure for Ylide Formation and Rearrangement}

To a solution of allyl sulfide compound ( $0.1 \mathrm{mmol}, 1.0$ equiv.) in 1,2-dimethoxyethane $(5 \mathrm{~mL})$, $\mathrm{Rh}_{2}(\mathrm{OAc})_{4}(0.005 \mathrm{mmol}, 0.05$ equiv.) was added, followed by addition of diazo compound in a nitrogen atmosphere. The reaction mixture was stirred vigorously at room temperature for 
$10 \mathrm{~h}$. Then another portion of diazo compound was added, and vigorous stirring was continued at room temperature for additional $12 \mathrm{~h}$. The solvent was removed by evaporation, and the remaining residue was purified by flash chromatography to afford the corresponding product.

$N$-(tert-Butoxycarbonyl)-S-(4-ethoxycarbonyl-1-buten-4-yl)-L-cysteine ethyl ester (3) was prepared according to the general procedure using 10 equiv. of ethyl diazoacetate. Colorless oil; ${ }^{1} \mathrm{H}$ NMR: $\delta$ 5.72-5.77 (m, 2H), 5.37 (br. d, $J=9.0 \mathrm{~Hz}, 1 \mathrm{H}$ ), 5.29 (br. d, $J=8.0$ $\mathrm{Hz}, 1 \mathrm{H}), 5.06-5.13$ (m, 4H), 4.54-4.55 (br. s, 2H), 4.16-4.23 (m, 8H), 3.40 (t, J=7.0 Hz, 1H), $3.32(\mathrm{dd}, J=8.5,6.5 \mathrm{~Hz}, 1 \mathrm{H}), 3.03-3.14(\mathrm{~m}, 3 \mathrm{H}), 2.91-2.95(\mathrm{~m}, 1 \mathrm{H}), 2.56-2.59(\mathrm{~m}, 2 \mathrm{H}), 2.38-$ $2.43(\mathrm{~m}, 2 \mathrm{H}), 1.44(\mathrm{~s}, 9 \mathrm{H}), 1.43(\mathrm{~s}, 9 \mathrm{H}), 1.27-1.29(\mathrm{~m}, 12 \mathrm{H}) .{ }^{13} \mathrm{C}$ NMR: $\delta 171.8,170.8,155.1$, 133.9, 133.8, 118.0, 80.1, 61.8, 61.7, 61.4, 61.3, 53.4, 53.0, 46.7, 46.2, 35.8, 35.5, 33.9, 28.3, 14.2. ESIHRMS Calcd for $\mathrm{C}_{17} \mathrm{H}_{29} \mathrm{NO}_{6} \mathrm{~S}[\mathrm{M}+\mathrm{Na}]^{+}: 398.1614$, found: 398.1607 .

$N$-(tert-Butoxycarbonyl)-S-(4-ethoxycarbonyl-2-methyl-1-buten-4-yl)-L-cysteine ethyl ester (4) was prepared according to the general procedure using 10 equiv. of ethyl diazoacetate. Colorless oil; ${ }^{1} \mathrm{H}$ NMR: $\delta 5.35$ (br. d, J = 8.1 Hz, 1H), 5.28 (br. d, $J=7.8 \mathrm{~Hz}, 1 \mathrm{H}$ ), 4.81 (s, 2H), 4.74 (d, $J=7.5,2 \mathrm{H}), 4.55$ (br. s, 2H), 4.15-4.23 (m, 8H), 3.47-3.59 (m, 2H), 3.06-3.15 (m, 4H), 2.92-2.95 (m, 2H), 2.56-2.61 (m, 2H), 2.31-2.35 (m, 2H), $1.74(\mathrm{~s}, 3 \mathrm{H}), 1.73(\mathrm{~s}, 3 \mathrm{H})$, 1.45 (s, 9H), 1.44 (s, 9H), 1.29-1.30 (m, 12H). ${ }^{13} \mathrm{C} \mathrm{NMR:} \delta$ 172.0, 170.8, 155.2, 141.5, 141.4, 113.1, 80.1, 61.8, 61.4, 61.3, 53.4, 53.0, 45.3, 44.9, 39.6, 39.4, 33.8, 28.3, 22.3, 14.2. ESIHRMS Calcd for $\mathrm{C}_{18} \mathrm{H}_{31} \mathrm{NO}_{6} \mathrm{~S}[\mathrm{M}+\mathrm{H}]^{+}: 390.1950$, found: 390.1944 .

$N$-(tert-Butoxycarbonyl)-S-(4-trimethylsilanyl-1-buten-4-yl)-L-cysteine ethyl ester (5) was prepared according to the general procedure using 5.0 equiv. of trimethylsilyl diazomethane. Colorless oil; ${ }^{1} \mathrm{H}$ NMR: $\delta$ 5.89-5.91 (m, 2H), 5.35 (br. d, $\left.J=8.0 \mathrm{~Hz}, 1 \mathrm{H}\right), 5.29$ (br. s, 1H), 5.02-5.11 (m, 4H), 4.48 (br. s, 2H), 4.17-4.22 (m, 4H), 2.86-3.03 (m, 6H), 2.48$2.50(\mathrm{~m}, 2 \mathrm{H}), 2.29-2.32(\mathrm{~m}, 2 \mathrm{H}), 1.92-1.93(\mathrm{~m}, 2 \mathrm{H}), 1.46(\mathrm{~s}, 9 \mathrm{H}), 1.44(\mathrm{~s}, 9 \mathrm{H}), 1.26-1.29$ (m, $6 \mathrm{H}), 0.15$ (s, 9H), 0.14 (s, 9H). ${ }^{13} \mathrm{C}$ NMR: $\delta 171.1,155.2,137.3,116.4,116.3,80.0,61.7,61.6$, 53.3, 53.2, 38.5, 36.2, 32.6, 32.0, 28.3, 14.2, -2.3. ESIHRMS Calcd for $\mathrm{C}_{17} \mathrm{H}_{33} \mathrm{NO}_{4} \mathrm{SSi}[\mathrm{M}$ $+\mathrm{Na}]^{+}:$398.1798, found: 398.1793.

$N$-(tert-Butoxycarbonyl)-S-(2-methyl-5-oxo-4-icosyl)-L-cysteine ethyl ester (6) was prepared according to the general procedure using 5.0 equiv. of 1-diazo-2-heptadecanone. Pale yellow oil; ${ }^{1} \mathrm{H}$ NMR: $\delta$ 5.27-5.30 (m, 2H) $4.81(\mathrm{~s}, 2 \mathrm{H}), 4.69(\mathrm{~s}, 2 \mathrm{H}), 4.49$ (br. s, 2H), 4.21 (q, $J=7.0 \mathrm{~Hz}, 4 \mathrm{H}), 3.45-3.48$ (m, 2H), 2.81-2.97 (m, 4H), 2.52-2.58 (m, 6H), 2.32-2.36 (m, 2H), $1.71(\mathrm{~s}, 6 \mathrm{H}), 1.44(\mathrm{~s}, 18 \mathrm{H}), 1.25-1.37(\mathrm{~m}, 58 \mathrm{H}), 0.87(\mathrm{t}, J=11.0 \mathrm{~Hz}, 6 \mathrm{H}) .{ }^{13} \mathrm{C} \mathrm{NMR:} \delta 206.3$, 206.2, 170.8, 155.1 141.6, 113.1, 80.2, 61.9, 53.2, 53.0, 51.0, 38.8, 38.1, 32.5, 29.7, 29.6, 29.4, 29.3, 29.2, 28.3, 23.9, 22.7, 22.5, 14.2. ESIHRMS Calcd for $\mathrm{C}_{31} \mathrm{H}_{57} \mathrm{NO}_{5} \mathrm{~S}[\mathrm{M}+\mathrm{H}]^{+}:$556.4036, found: 556.4027 .

$N$-(tert-Butoxycarbonyl)-S-(4-ethoxycarbonyl-1-buten-4-yl)-glutathione dimethyl ester (8) was prepared according to the general procedure using 10 equiv. of ethyl diazoacetate. Pale yellow oil; ${ }^{1} \mathrm{H}$ NMR: $\delta 7.16$ (br. d, $J=5.0 \mathrm{~Hz}, 2 \mathrm{H}$ ), 6.99 (br. d, $J=6.5 \mathrm{~Hz}, 1 \mathrm{H}$ ), 6.89 (br. d, $J=6.0 \mathrm{~Hz}, 1 \mathrm{H}), 5.72-5.75(\mathrm{~m}, 2 \mathrm{H}), 5.35$ (br. d, $J=5.5 \mathrm{~Hz}, 2 \mathrm{H}), 5.09-5.14(\mathrm{~m}, 4 \mathrm{H}), 4.62-4.67$ $(\mathrm{m}, 2 \mathrm{H}), 4.32-4.38(\mathrm{~m}, 2 \mathrm{H}), 4.18-4.24(\mathrm{~m}, 4 \mathrm{H}), 4.01-4.09(\mathrm{~m}, 4 \mathrm{H}), 3.74(\mathrm{~s}, 6 \mathrm{H}), 3.73(\mathrm{~s}, 6 \mathrm{H})$, $3.52(\mathrm{t}, J=7.3 \mathrm{~Hz}, 1 \mathrm{H}), 3.44(\mathrm{t}, J=7.5 \mathrm{~Hz}, 1 \mathrm{H}), 3.19(\mathrm{dd}, J=14.3,6.3 \mathrm{~Hz}, 2 \mathrm{H}), 2.94-3.02$ (m, 2H), 2.59-2.65 (m,2H), 2.44-2.48 (m, 2H), 2.32-2.38 (m, 4H), 2.16-2.21 (m, 2H), 1.93$2.01(\mathrm{~m}, 2 \mathrm{H}), 1.43(\mathrm{~s}, 18 \mathrm{H}), 1.24-1.29(\mathrm{~m}, 6 \mathrm{H}) .{ }^{13} \mathrm{C}$ NMR: $\delta 172.9,172.6,172.3,170.4,169.9$, $155.7,133.8,118.1,80.2,61.7,52.6,47.2,41.3,35.7,33.5,33.0,32.1,28.6,14.2$. ESIHRMS Calcd for $\mathrm{C}_{24} \mathrm{H}_{39} \mathrm{~N}_{3} \mathrm{O}_{10} \mathrm{~S}[\mathrm{M}+\mathrm{Na}]^{+}: 584.2254$, found: 584.2255 .

$N$-(tert-Butoxycarbonyl)-S-(4-phenyl-1-buten-4-yl)-glutathione dimethyl ester (9) was prepared according to the general procedure using 5.0 equiv. of phenyl diazomethane. Pale yellow oil; ${ }^{1} \mathrm{H}$ NMR: $\delta 7.31-7.32(\mathrm{~m}, 4 \mathrm{H}), 7.22-7.25$ (m, 6H), 7.02 (br. s, $\left.1 \mathrm{H}\right), 6.82$ (br. s, 
$1 \mathrm{H}$ ), 6.67 (br. d, $J=6.5 \mathrm{~Hz}, 1 \mathrm{H}) 6.60$ (br. d, $J=7.0 \mathrm{~Hz}, 1 \mathrm{H}$ ), $5.63-5.70$ (m, $2 \mathrm{H}$ ), 5.37 (br. s, 2H), 4.95-5.04 (m, 4H), 4.32-4.45 (m, 4H), 3.86-3.98 (m, 8H), $3.73(\mathrm{~s}, 6 \mathrm{H}), 3.72(\mathrm{~s}, 6 \mathrm{H}), 2.83$ (dd, $J=12.0,5.8 \mathrm{~Hz}, 1 \mathrm{H}), 2.69-2.71(\mathrm{~m}, 2 \mathrm{H}), 2.58-2.64(\mathrm{~m}, 5 \mathrm{H}), 2.27-2.31(\mathrm{~m}, 2 \mathrm{H}), 2.21-$ $2.24(\mathrm{~m}, 2 \mathrm{H}), 2.11-2.18(\mathrm{~m}, 2 \mathrm{H}), 1.89-1.96(\mathrm{~m}, 2 \mathrm{H}), 1.42(\mathrm{~s}, 9 \mathrm{H}), 1.41$ (s, 9H). ${ }^{13} \mathrm{C}$ NMR $(125$ $\left.\mathrm{MHz}, \mathrm{CDCl}_{3}\right) \delta 172.9,172.2,172.1,170.6,170.0,169.9,155.7,142.0,141.9,135.1,135.0$, $128.7,127.9,127.5,117.3,80.2,52.8,52.5,52.4,52.2,50.2,50.0,49.9,41.3,40.7,40.6,32.8$, 32.2, 28.7, 28.5, 28.3. ESIHRMS Calcd for $\mathrm{C}_{27} \mathrm{H}_{39} \mathrm{~N}_{3} \mathrm{O}_{8} \mathrm{~S}[\mathrm{M}+\mathrm{Na}]^{+}$: 588.2356, found: 588.2364 .

$N$-(tert-Butoxycarbonyl)-S-(5-oxo-4-icosyl)-glutathione dimeth-yl ester (10) was prepared according to the general procedure using 5.0 equiv. of 1-diazo-2-heptadecanone. Pale yellow oil; ${ }^{1} \mathrm{H}$ NMR: $\delta 7.11-7.13$ (br. s, $2 \mathrm{H}$ ), 6.81 (br. d, $J=6.0 \mathrm{~Hz}, 1 \mathrm{H}$ ), 6.80 (br. d, $J=6.5 \mathrm{~Hz}, 1 \mathrm{H}$ ), 5.69-5.77 (m, 2H), 5.34-5.35 (br. d, $J=7.0 \mathrm{~Hz}, 2 \mathrm{H}), 5.07-5.09(\mathrm{~m}, 4 \mathrm{H}), 4.55-4.59(\mathrm{~m}, 2 \mathrm{H})$, 4.36 (br. s, 2H), 3.95-4.09 (m, 4H), $3.74(\mathrm{~s}, 12 \mathrm{H}), 3.44-3.49(\mathrm{~m}, 2 \mathrm{H}), 2.80-2.90(\mathrm{~m}, 4 \mathrm{H}), 2.55-$ $2.62(\mathrm{~m}, 6 \mathrm{H}), 2.32-2.46(\mathrm{~m}, 6 \mathrm{H}), 2.14-2.17(\mathrm{~m}, 2 \mathrm{H}), 1.91-1.97(\mathrm{~m}, 2 \mathrm{H}), 1.51-1.57(\mathrm{~m}, 4 \mathrm{H})$, $1.42(\mathrm{~s}, 18 \mathrm{H}), 1.16-1.28(\mathrm{~m}, 48 \mathrm{H}), 0.85-0.88(\mathrm{t}, J=7.0 \mathrm{~Hz}, 6 \mathrm{H}) .{ }^{13} \mathrm{C}$ NMR: $\delta 207.7,207.3$, $172.9,172.3,172.2,170.4,170.3,169.9,155.7,134.2,134.1,118.1,118.0,80.2,52.8,52.7$, 52.6, 52.5, 52.4, 52.2, 41.3, 39.7, 39.6, 34.5, 32.4, 32.2, 32.1,31.9, 29.7, 29.6, 29.5, 29.4, 29.3, 29.2, 28.7, 28.5, 28.3, 23.9, 22.7, 14.1. ESIHRMS Calcd for $\mathrm{C}_{37} \mathrm{H}_{65} \mathrm{~N}_{3} \mathrm{O}_{9} \mathrm{~S}[\mathrm{M}+\mathrm{Na}]^{+}$: 750.4340, found: 750.4322 .

$N$-(tert-Butoxycarbonyl)-S-(1-tetradecen-4-yl)-glutathione dimethyl ester (11) was prepared according to the general procedure using 5.0 equiv. of 1-diazo-undecane. Pale yellow oil; ${ }^{1} \mathrm{H}$ NMR: $\delta 7.16$ (br. d, $\left.J=5.0 \mathrm{~Hz}, 2 \mathrm{H}\right), 6.84$ (br. d, $\left.J=7.0 \mathrm{~Hz}, 2 \mathrm{H}\right), 5.81-5.84(\mathrm{~m}, 2 \mathrm{H}$ ), 5.33 (br. d, $J=6.5 \mathrm{~Hz}, 2 \mathrm{H}$ ), $5.05-5.10(\mathrm{~m}, 4 \mathrm{H}), 4.52-4.53$ (m, 2H), 4.39 (br. d, $J=4.5 \mathrm{~Hz}$, 2H), 3.98-4.05 (m, 4H), $3.74(\mathrm{~s}, 12 \mathrm{H}), 2.97-2.98(\mathrm{~m}, 2 \mathrm{H}), 2.76-2.79(\mathrm{~m}, 4 \mathrm{H}), 2.34-2.38(\mathrm{~m}$, $8 \mathrm{H}), 2.11-2.19(\mathrm{~m}, 2 \mathrm{H}), 1.85-1.96(\mathrm{~m}, 2 \mathrm{H}), 1.51-1.54(\mathrm{~m}, 2 \mathrm{H}), 1.43(\mathrm{~s}, 18 \mathrm{H}), 1.24-1.39(\mathrm{~m}$, 34H), 0.85-0.88 (m, 6H). ${ }^{13} \mathrm{C}$ NMR: $\delta$ 172.9, 172.1, 170.7, 169.9, 155.7, 135.6, 135.5, 117.3, 117.2, 80.2, 52.8, 52.7, 52.5, 52.4, 46.3, 45.9, 41.3, 39.1, 34.6, 34.2, 32.3, 32.1, 31.9, 29.6, 29.5, 29.4, 28.6, 28.3, 26.8, 26.7, 22.7, 14.1. ESIHRMS Calcd for $\mathrm{C}_{31} \mathrm{H}_{55} \mathrm{~N}_{3} \mathrm{O}_{8} \mathrm{~S}[\mathrm{M}+\mathrm{H}]^{+}$: 630.3788, found: 630.3773 .

$N$-(tert-Butoxycarbonyl)-S-(1-tridecafluorododecen-4-yl)-glutathione dimethyl ester (12) was prepared according to the general procedure using 5.0 equiv. of 9diazo-1,1,1,2,2,3,3,4,4,5,-5,6,6-tridecafluorononane. Pale yellow oil; ${ }^{1} \mathrm{H}$ NMR: $\delta 7.11$ (br. s, $2 \mathrm{H}), 6.88$ (br. s, 2H), 5.74-5.77 (m, 2H), 5.45 (br. s, 2H), 5.33-5.36 (m, 2H), 5.12-5.18 (m, $4 \mathrm{H}), 4.57-4.58(\mathrm{~m}, 1 \mathrm{H}), 4.31-4.42(\mathrm{~m}, 2 \mathrm{H}), 3.98-4.13(\mathrm{~m}, 4 \mathrm{H}), 3.75(\mathrm{~s}, 12 \mathrm{H}), 3.29-3.32(\mathrm{~m}$, $2 \mathrm{H}), 3.04-3.23(\mathrm{~m}, 4 \mathrm{H}), 2.74-2.95(\mathrm{~m}, 4 \mathrm{H}), 2.33-2.51(\mathrm{~m}, 4 \mathrm{H}), 2.17-2.20(\mathrm{~m}, 2 \mathrm{H}), 1.89-2.16$ $(\mathrm{m}, 4 \mathrm{H}), 1.44$ (s, 9H), 1.45 (s, 9H). ${ }^{13} \mathrm{C}$ NMR: $\delta 172.8,171.9,171.4,169.9,155.6,133.4,133.2$, $118.4,118.2,80.7,52.9,52.7,52.5,45.8,41.4,39.5,34.9,34.3,32.4,32.2,31.7,29.7,29.3$, 28.8, 28.3,28.2, 27.8, 24.6. $\mathrm{F}^{19} \mathrm{NMR}: \delta-8.3,-41.7,-49.5,-50.5,-50.9,-53.7$. ESIHRMS Calcd for $\mathrm{C}_{29} \mathrm{H}_{38} \mathrm{~F}_{13} \mathrm{~N}_{3} \mathrm{O}_{8} \mathrm{~S}[\mathrm{M}+\mathrm{H}]^{+}: 836.2245$, found: 836.2247.

$\boldsymbol{N}$-(2,3,4,6-Tetra- $\boldsymbol{O}$-acetyl- $\boldsymbol{\beta}$-D-glucopyranosyl)-(2-hexadecylsulfanyl)-4-pentenamide (16) was prepared according to the general procedure using 5.0 equiv. of $\mathrm{N}$-(2,3,4,6-tetra- $O$ acetyl- $\beta$-D-glucopyranosyl)-diazoacetamide. Colorless oil; ${ }^{1} \mathrm{H}$ NMR: $\delta 7.21-7.30(\mathrm{~m}, 2 \mathrm{H})$, $5.66-5.79(\mathrm{~m}, 2 \mathrm{H}), 5.30(\mathrm{t}, J=9.5 \mathrm{~Hz}, 2 \mathrm{H}), 5.19-5.23(\mathrm{~m}, 2 \mathrm{H}), 5.01-5.33(\mathrm{~m}, 6 \mathrm{H}), 4.96-5.00$ $(\mathrm{m}, 2 \mathrm{H}), 4.28-4.32(\mathrm{~m}, 2 \mathrm{H}), 4.05-4.08(\mathrm{~m}, 2 \mathrm{H}), 3.79-3.82(\mathrm{~m}, 2 \mathrm{H}), 3.25-3.28(\mathrm{~m}, 2 \mathrm{H}), 2.38-$ $2.49(\mathrm{~m}, 6 \mathrm{H}), 2.17(\mathrm{~s}, 6 \mathrm{H}), 2.07(\mathrm{~s}, 6 \mathrm{H}), 2.03(\mathrm{~s}, 6 \mathrm{H}), 2.02(\mathrm{~s}, 6 \mathrm{H}), 1.52-1.55(\mathrm{~m}, 4 \mathrm{H}), 1.24$ $1.33(\mathrm{~m}, 54 \mathrm{H}), 0.87(\mathrm{t}, J=7.0 \mathrm{~Hz}, 6 \mathrm{H}) .{ }^{13} \mathrm{C}$ NMR: $\delta 172.5,172.4,170.6,170.4,170.0,169.6$, 133.9, 133.8, 118.2, 117.9, 78.4, 73.6, 72.9, 72.8, 70.3, 68.2, 61.7, 49.5, 49.4, 36.5, 36.2, 31.9, 31.5, 31.4, 29.7, 29.6, 29.5, 29.4, 29.2, 29.0, 28.9, 22.7, 20.7, 20.6, 14.2. ESIHRMS Calcd for $\mathrm{C}_{35} \mathrm{H}_{59} \mathrm{~N}_{3} \mathrm{O}_{10} \mathrm{~S}[\mathrm{M}+\mathrm{Na}]^{+}:$708.3758, found: 708.3732 . 
$N$-(tert-Butoxycarbonyl)-S-(4- $N$-(2,3,4,6-Tetra- $O$-acetyl- $\beta$-D-glucopyranosyl) aminocarbonyl-1-buten-4-yl)-L-cysteine ethyl ester (17) was prepared according to the general procedure using 5.0 equiv. of $N$-(2,3,4,6-tetra- $O$-acetyl- $\beta$-D-glucopyranosyl) diazoacetamide. Pale yellow oil; ${ }^{1} \mathrm{H}$ NMR: $\delta 7.41$ (br. d, $J=8.5 \mathrm{~Hz}, 1 \mathrm{H}$ ), 7.27 (br. s, $1 \mathrm{H}$ ), 5.55$5.74(\mathrm{~m}, 2 \mathrm{H}), 5.64-5.74(\mathrm{~m}, 1 \mathrm{H}), 5.50$ (br. d, $J=7.5 \mathrm{~Hz}, 1 \mathrm{H}), 5.19-5.30(\mathrm{~m}, 4 \mathrm{H}), 4.98-5.12$ (m, 6H), 4.43 (br, 2H), 4.25-4.30 (m, 2H), 4.15-4.21 (m, 4H), 4.02-4.11 (m, 4H), 3.77-3.81 (m, 2H), 3.30-3.33 (m, 2H), 3.09 (br. d, $J=13.0 \mathrm{~Hz}, 1 \mathrm{H}), 2.94-2.97(\mathrm{~m}, 1 \mathrm{H}), 2.68-2.74(\mathrm{~m}$, $2 \mathrm{H}), 2.60-2.65(\mathrm{~m}, 1 \mathrm{H}), 2.48-2.54(\mathrm{~m}, 1 \mathrm{H}), 2.33-2.40(\mathrm{~m}, 2 \mathrm{H}), 1.98-2.05(\mathrm{~m}, 24 \mathrm{H}), 1.47(\mathrm{~s}$, 9H), 1.46 (s, 9H), $1.21-1.27(\mathrm{~m}, 6 \mathrm{H}) .{ }^{13} \mathrm{C}$ NMR: $\delta 171.8,171.6,171.2,170.7,170.6,170.4$, 169.9, 169.5, 169.4, 155.5, 155.4, 133.9, 133.4, 118.2, 118.1, 80.5, 80.4, 73.6, 72.9, 72.7, 70.3, 68.1, 68.0, 62.0, 61.9, 61.7, 61.5, 60.4, 53.0, 49.8, 48.0, 36.2, 35.8, 34.2, 33.7, 28.4, 21.1, 20.7, 20.6, 14.2. ESIHRMS Calcd for $\mathrm{C}_{29} \mathrm{H}_{44} \mathrm{~N}_{2} \mathrm{O}_{14} \mathrm{~S}[\mathrm{M}+\mathrm{Na}]^{+}$: 699.2406, found: 699.2410.

$N$-(tert-Butoxycarbonyl)-S-(4- $N$-(2,3,6,2', $3^{\prime}, 4^{\prime}, 6^{\prime}$-hepta- $O$-acetyl- $\beta$-cellobiosyl) aminocarbonyl-1-buten-4-yl)-L-cysteine ethyl ester (18) was prepared according to the general procedure using 5.0 equiv. of $N$ - $\left(2,3,6,2^{\prime}, 3^{\prime}, 4^{\prime}, 6^{\prime}\right.$-hepta- $O$-acetyl- $\beta$-cellobiosyl $)$ diazoacetamide. Pale yellow oil; ${ }^{1} \mathrm{H}$ NMR: $\delta 7.26$ (br. d, $J=9.0 \mathrm{~Hz}, 1 \mathrm{H}$ ), 7.13 (br. d, $J=9.0$ $\mathrm{Hz}, 1 \mathrm{H}), 5.59-5.61(\mathrm{~m}, 2 \mathrm{H}), 5.46$ (br. d, $J=6.6 \mathrm{~Hz}, 1 \mathrm{H}), 5.21-5.28(\mathrm{~m}, 3 \mathrm{H}), 5.03-5.19(\mathrm{~m}$, $10 \mathrm{H}), 4.88-4.92(\mathrm{~m}, 4 \mathrm{H}), 4.40-4.49(\mathrm{~m}, 6 \mathrm{H}), 4.33-4.35(\mathrm{~m}, 2 \mathrm{H}), 4.08-4.19(\mathrm{~m}, 8 \mathrm{H}), 3.98-$ $4.01(\mathrm{~m}, 2 \mathrm{H}), 3.62-3.71(\mathrm{~m}, 6 \mathrm{H}), 3.26-3.31(\mathrm{~m}, 2 \mathrm{H}), 3.03-3.08(\mathrm{~m}, 1 \mathrm{H}), 2.91-2.96(\mathrm{~m}, 1 \mathrm{H})$, 2.68-2.73 (m, 1H), 2.59-2.66 (m, 1H), 2.45-2.51 (m, 1H), 2.31-2.39 (m, 2H), 1.95-2.09 (m, $42 \mathrm{H}), 1.45$ (s, 9H), $1.42(\mathrm{~s}, 9 \mathrm{H}), 1.21-1.27(\mathrm{~m}, 6 \mathrm{H}) .{ }^{13} \mathrm{C}$ NMR: $\delta$ 171.6, 171.4, 170.8, 170.7, 170.6, 170.5, 170.2, 169.4, 169.3, 169.0, 155.3, 133.7, 133.4, 118.2, 118.1, 100.7, 80.378.3, $78.2,76.2,74.5,72.9,72.2,72.1,72.0,71.5,70.5,67.8,62.0,61.9,61.6,60.4,53.5,53.1,49.8$, 48.4, 36.2, 35.8, 34.1, 33.5, 28.4, 21.1, 20.8, 20.7, 20.5, 14.2. ESIHRMS Calcd for $\mathrm{C}_{41} \mathrm{H}_{60} \mathrm{~N}_{2} \mathrm{O}_{22} \mathrm{~S}[\mathrm{M}+\mathrm{H}]^{+}$: 965.3431 , found: 965.3424 .

\section{$N$-(tert-Butoxycarbonyl)-S-(4- $N$-(2,3,4,6-Tetra- $O$-acetyl- $\beta$-D-glucopyranosyl)} aminocarbonyl-1-buten-4-yl)-glutathione dimethyl ester (19) was prepared according to the general procedure using 5.0 equiv. of $N$-(2,3,4,6-tetra- $O$-acetyl- $\beta$-D-glucopyranosyl)diazoacetamide. Pale yellow oil; ${ }^{1} \mathrm{H}$ NMR: $\delta 7.69$ (br. d, $J=8.5 \mathrm{~Hz}, 2 \mathrm{H}$ ), 7.35 (br. s, 2H), 7.01 (br. d, $J=7.5 \mathrm{~Hz}, 2 \mathrm{H}), 5.73-5.76(\mathrm{~m}, 2 \mathrm{H}), 5.35$ (br. s, 2H), 5.26-5.31 (m, 4H), 4.99-5.14 (m, $6 \mathrm{H}), 4.82$ (br. s, $2 \mathrm{H}), 4.32-4.40(\mathrm{~m}, 4 \mathrm{H}), 4.03-4.14(\mathrm{~m}, 4 \mathrm{H}), 3.82-3.87(\mathrm{~m}, 2 \mathrm{H}), 3.73-3.79$ (m, 12H), 3.63 (br. s, 2H), 2.96-3.31 (m, 4H), 2.62-2.64 (m, 2H), 2.52-2.54 (m, 2H), 2.352.37 (m, 4H), 2.00-2.19 (m, 28H), 1.43 (s, 9H), 1.42 (s, 9H). ${ }^{13} \mathrm{C}$ NMR: $\delta 172.9,172.3,172.1$, 170.8, 170.6, 170.3, 170.0, 169.6, 155.6, 133.9, 133.8, 118.2, 80.2, 78.3, 73.9, 73.1, 70.6, 70.5, 68.2, 68.1, 61.7, 60.4, 52.8, 52.6, 52.1, 52.0, 49.2, 45.4, 41.3, 41.2, 36.0, 35.2, 33.8, 32.6, 31.9, 28.3, 24.0, 21.0, 20.8. ESIHRMS Calcd for $\mathrm{C}_{36} \mathrm{H}_{54} \mathrm{~N}_{4} \mathrm{O}_{18} \mathrm{~S}[\mathrm{M}+\mathrm{H}]^{+}:$885.3046, found: 885.3059.

$N$-(tert-Butoxycarbonyl)-S-(4- $N$-(2,3,6, $2^{\prime}, 3^{\prime}, 4^{\prime}, 6^{\prime}$-hepta- $O$-acetyl- $\beta$-cellobiosyl) aminocarbonyl-1-buten-4-yl)-glutathione dimethyl ester (20) was prepared according to the general procedure using 5.0 equiv. of $N$ - $\left(2,3,6,2^{\prime}, 3^{\prime}, 4^{\prime}, 6^{\prime}\right.$-hepta- $O$-acetyl- $\beta$-cellobiosyl $)$ diazoacetamide. Pale yellow oil; ${ }^{1} \mathrm{H}$ NMR: $\delta 7.55$ (br. d, $J=9.1 \mathrm{~Hz}, 1 \mathrm{H}$ ), 7.28 (br. d, J $=7.0$ $\mathrm{Hz}, 1 \mathrm{H}), 6.98$ (br. d, $J=8.1 \mathrm{~Hz}, 1 \mathrm{H}), 5.62-5.71(\mathrm{~m}, 1 \mathrm{H}), 5.33$ (br. d, $J=8.1 \mathrm{~Hz}, 1 \mathrm{H}), 5.20-$ $5.27(\mathrm{~m}, 4 \mathrm{H}), 5.10-5.14(\mathrm{~m}, 3 \mathrm{H}), 5.03-5.07(\mathrm{~m}, 4 \mathrm{H}), 4.89-4.92(\mathrm{~m}, 4 \mathrm{H}), 4.61-4.68$ (br. s, $1 \mathrm{H})$, 4.48-4.52 (m, 5H), 4.34-4.37 (m, 2H), 4.25 (br. s, 1H), 4.03-4.12 (m, 8H), 3.71-3.76 (m, $10 \mathrm{H}), 3.64-3.66$ (m, 3H), 3.12 (br. s, 1H), 3.01 (br. s, $1 \mathrm{H}), 2.52-2.61(\mathrm{~m}, 1 \mathrm{H}), 2.50-2.51(\mathrm{~m}$, 1H), 2.35-2.37 (m, 2H), 1.98-2.13 (m, 21H), $1.42(\mathrm{~s}, 9 \mathrm{H}) .{ }^{13} \mathrm{C}$ NMR: $\delta$ 173.0, 172.3, 171.5, $170.6,170.5,170.3,170.2,169.9,169.8,169.3,169.0,155.6,133.9,133.8,118.0,100.6,80.3$, 78.1, 76.4, 74.9, 73.2, 73.1, 71.9, 71.8, 71.6, 70.5, 67.8, 61.8, 61.7, 61.5, 52.9, 52.6, 52.5, 52.4, 46.4, 41.4, 35.1, 32.8, 32.0, 28.3, 20.8. ESIHRMS Calcd for $\mathrm{C}_{48} \mathrm{H}_{70} \mathrm{~N}_{4} \mathrm{O}_{26} \mathrm{~S}[\mathrm{M}+\mathrm{H}]^{+}$: 1151.4072, found: 1151.4071 . 
$N$-(tert-Butoxycarbonyl)-S-(2-methyl-5-oxo-4-icosyl)-L-cysteinyl-L-alanyl-Ltryptophan methyl ester (21) was prepared according to the general procedure using 5.0 equiv. of 1-diazo-2-heptadecanone, which was contaminated by approximately $10 \%$ of 22. ${ }^{1} \mathrm{H}$ NMR: $\left(400 \mathrm{MHz}, \mathrm{CDCl}_{3}\right) \delta 8.68(\mathrm{~s}, 1 \mathrm{H}), 8.59(\mathrm{~s}, 1 \mathrm{H}), 7.51(\mathrm{~d}, J=7.7 \mathrm{~Hz}, 1 \mathrm{H}), 7.50$ $(\mathrm{d}, J=7.7 \mathrm{~Hz}, 1 \mathrm{H}), 7.34(\mathrm{~m}, 2 \mathrm{H}) 7.16(\mathrm{~m}, 2 \mathrm{H}), 7.09(\mathrm{~m}, 2 \mathrm{H}), 7.00(\mathrm{~s}, 1 \mathrm{H}), 6.98(\mathrm{~s}, 1 \mathrm{H}), 6.80$ $6.67(\mathrm{~m}, 4 \mathrm{H}), 5.28(\mathrm{~m}, 1 \mathrm{H}), 5.15(\mathrm{~m}, 1 \mathrm{H}), 4.89-4.80(\mathrm{~m}, 4 \mathrm{H}), 4.73(\mathrm{~s}, 1 \mathrm{H}), 4.70(\mathrm{~s}, 1 \mathrm{H})$, 4.47-4.37 (m, 2H), 4.20-4.10 (m, 2H), $3.694(\mathrm{~s}, 3 \mathrm{H}), 3.692(\mathrm{~s}, 3 \mathrm{H}), 3.53(\mathrm{~m}, 2 \mathrm{H}), 3.37-3.23$ (m, 4H), 2.74-2.73 (m, 4H), 2.62-2.50 (m, 6H), 2.38-2.31 (m, 2H), $1.74(\mathrm{~s}, 3 \mathrm{H}), 1.73(\mathrm{~s}, 3 \mathrm{H}$,$) ,$ $1.63-1.56(\mathrm{~m}, 4 \mathrm{H}), 1.47$ (s, 18H), 1.31 (d, $J=7.1 \mathrm{~Hz}, 6 \mathrm{H}), 1.30-1.20(\mathrm{~m}, 52 \mathrm{H}), 0.88$ (t, $J=7.0$ $\mathrm{Hz}, 6 \mathrm{H}) .{ }^{13} \mathrm{C} \mathrm{NMR}\left(100 \mathrm{MHz}, \mathrm{CDCl}_{3}\right) \delta 207.6,207.4,172.04,171.99,171.1,170.13,170.07$, 155.6, 141.6, 141.5, 136.3, 127.5, 127.4, 123.23, 123.19, 122.06, 122.04, 113.3, 111.38, 111.33, 109.5, 79.8, 54.0, 53.4, 53.1, 52.7, 52.4, 50.9, 50.8, 48.9, 39.1, 39.0, 38.0, 37.9, 32.4, 32.2, 31.9, 29.68, 29.65, 29.5, 29.4, 29.3, 29.2, 28.3, 27.4, 27.3, 23.9, 22.7, 22.4, 17.5, 17.4, 14.1. ESIHRMS Calcd for $\mathrm{C}_{44} \mathrm{H}_{71} \mathrm{~N}_{4} \mathrm{O}_{7} \mathrm{~S}$ [M+H] $]^{+}: 799.5038$, found: 799.5038; Calcd for $\mathrm{C}_{44} \mathrm{H}_{70} \mathrm{~N}_{4} \mathrm{O}_{7} \mathrm{SNa}[\mathrm{M}+\mathrm{Na}]^{+}$: 821.4858, found: 821.4560.

$N$-(tert-Butoxycarbonyl)-S-(2-methylallyl)-L-cysteinyl-L-alanyl-L- $N_{\omega}$-(2-oxo-

heptadecyl)-tryptophan methyl ester (22) was prepared according to the general procedure using 5.0 equiv. of 1-diazo-2-heptadecanone. ${ }^{1} \mathrm{H} \mathrm{NMR}\left(400 \mathrm{MHz}, \mathrm{CDCl}_{3}\right) \delta 7.50(\mathrm{~d}, J=7.7$ $\mathrm{Hz}, 1 \mathrm{H}), 7.20(\mathrm{~m}, 1 \mathrm{H}), 7.14-7.10(\mathrm{~m}, 2 \mathrm{H}), 6.90(\mathrm{~s}, 1 \mathrm{H}), 6.84$ (br. d, J = 7.7 Hz, 1H), 6.59 (br. $\mathrm{d}, J=7.6 \mathrm{~Hz}, 1 \mathrm{H}), 5.29(\mathrm{~m}, 1 \mathrm{H}), 4.93-4.79(\mathrm{~m}, 4 \mathrm{H}), 4.45$ (quint, $J=7.4 \mathrm{~Hz}, 1 \mathrm{H}), 4.14(\mathrm{~m}, 1 \mathrm{H})$, $3.68(\mathrm{~s}, 3 \mathrm{H}), 3.33(\mathrm{~d}, J=5.6 \mathrm{~Hz}, 2 \mathrm{H}), 3.03(\mathrm{~d}, J=13.4 \mathrm{~Hz}, 1 \mathrm{H}), 2.96(\mathrm{~d}, J=13.4 \mathrm{~Hz}, 1 \mathrm{H})$, $2.72(\mathrm{dd}, J=13.7,5.9 \mathrm{~Hz}, 1 \mathrm{H}), 2.65(\mathrm{dd}, J=13.7,6.7 \mathrm{~Hz}, 1 \mathrm{H}), 2.35(\mathrm{t}, J=7.4 \mathrm{~Hz}, 2 \mathrm{H}), 1.77$ (s, 3H), $1.56(\mathrm{~m}, 2 \mathrm{H}), 1.44(\mathrm{~s}, 9 \mathrm{H}), 1.32(\mathrm{~d}, J=7.1 \mathrm{~Hz}, 3 \mathrm{H}), 1.30-1.20(\mathrm{~m}, 26 \mathrm{H}), 0.88(\mathrm{~d}, J=$ $7.0 \mathrm{~Hz}, 3 \mathrm{H}) .{ }^{13} \mathrm{C} \mathrm{NMR}\left(100 \mathrm{MHz}, \mathrm{CDCl}_{3}\right) \delta 206.4,171.8,171.3,170.4,140.7,136.6,128.1$, 127.6, 122.4, 119.8, 119.0, 114.5, 109.6, 108.9, 80.4, 55.2, 53.8, 52.6, 52.5, 48.9, 39.4, 33.1, 31.9, 29.7, 29.5, 29.4, 29.1, 28.3, 27.3, 23.4, 22.7, 20.6, 18.0, 14.1. FABHRMS Calcd for $\mathrm{C}_{44} \mathrm{H}_{70} \mathrm{~N}_{4} \mathrm{O}_{7} \mathrm{SNa}[\mathrm{M}+\mathrm{Na}]^{+}$: 821.4863, found: 821.4859.

\section{Supplementary Material}

Refer to Web version on PubMed Central for supplementary material.

\section{Acknowledgements}

We thank the NIH (GM 62160) for partial support of this work.

\section{References}

1. (a) Crich D, Krishnamurthy V, Hutton TK. J Am Chem Soc 2006;128:2544. [PubMed: 16492032] (b) Crich D, Brebion F, Krishnamurthy V. Org Lett 2006;8:3593. [PubMed: 16869668]

2. Trost, BM.; Melvin, SM. Sulfur Ylides. Academic Press; New York: 1975. (b) Vedejs E. Acc Chem Res 1984;17:358.

3. (a) Kirmse W, Kapps M. Chem Ber 1968;101:994. (b) Doyle MP, Tamblyn WH, Bagheri V. J Org Chem 1981;46:4094. (c) Doyle MP, Griffin JH, Chinn MS, van Leusen D. J Org Chem 1984;49:1917. (d) Doyle MP, Tamblyn WH, Bagheri V. J Org Chem 1981;46:5094. (e) Ando W, Yagihara T, Kondo S, Nakayama K, Yamato H, Nakaido S, Migita T. J Org Chem 1971;36:1732.Doyle, MP.; McKervey, MA.; Ye, T. Modern Catalytic Methods for Organic Synthesis with Diazo compounds. Wiley; New York: 1998. Aggarwal, V.; Richardson, J. Science of Synthesis: Compounds with Two CarbonHeteroatom Bonds. Padwa, A., editor. 27. Thieme; Stuttgart: 2004. p. 21 (h) Li AH, Dai LX, Aggarwal VK. Chem Rev 1997;97:2341. [PubMed: 11848902] (i) Doyle MP, Forbes DC. Chem Rev 1998;98:911. [PubMed: 11848918] (j) Hodgson DM, Pierard FYTM, Stupple PA. Chem Soc Rev 2001;30:50. 
4. (a) McFarland JM, Francis MF. J Am Chem Soc 2005;127:13490. [PubMed: 16190700] (b) Tilley DS, Francis MB. J Am Chem Soc 2006;128:1080. [PubMed: 16433516] (c) van Maarseveen JH, Reek JNH, Back JW. Angew Chem Int Ed 2006;45:1841. (d) Dibowski H, Schmidtchen FP. Angew Chem Int Ed 1998;37:476. (e) Antos JM, Francis MB. Curr Op Chem Biol 2006;10:253.

5. Antos JM, Francis MF. J Am Chem Soc 2004;126:10256. [PubMed: 15315433]

6. (a) Carter DS, Van Vranken DL. Tetrahedron Lett 1999;40:1617. (b) Aggarwal VK, Ferrara M, Hainz R, Spey SE. Tetrahedron Lett 1999;40:8923.

7. (a) Pachamuthu K, Schmidt RR. Chem Rev 2006;106:160. [PubMed: 16402775] (b) Clarke S. Ann Rev Biochem 1992;61:355. [PubMed: 1497315] (c) Schelhaas M, Nägele E, Kuder N, Bader B, Kuhlmann J, Wittinghofer A, Waldmann H. Chem Eur J 1999;5:1239. (d) Schelhaas M, Glomsda S, Hänsler M, Jakubke HD, Waldmann H. Angew Chem Int Ed 1996;35:106. (e) Brown MJ, Milano PD, Lever DC, Epstein WW, Poulter CD. J Am Chem Soc 1991;113:3176. (f) Pachamuthu K, Zhu X, Schmidt RR. J Org Chem 2005;70:3720. [PubMed: 15845014]

8. Brittain SM, Ficarro SB, Brock A, Peters EC. Nature Biotechnology 2005;23:463.

9. (a) Gamblin DP, Garnier P, van Kasteren S, Oldham NJ, Fairbanks AJ, Davis BG. Angew Chem Int Ed 2004;43:828. (b) Gamblin DP, Garnier SJ, Ward NJ, Oldham AJ, Fairbanks AJ, Davis BG. Org Biomol Chem 2003;1:3642. [PubMed: 14649893] (c) Doores KJ, Gamblin DP, Davis BG. Chem Eur J 2006;12:656. (d) Davis BG. Chem Rev 2002;102:579. [PubMed: 11841255] (e) Peri F, Nicotra F. Chem Comm 2004:623. (f) Ohnishi Y, Ichikawa M, Ichikawa Y. Biorg Med Chem Lett 2000;10:1289. (g) Jobron L, Hummel G. Org Lett 2000;2:2265. [PubMed: 10930259] (h) Cohen SB, Halcomb RL. Org Lett 2001;3:405. [PubMed: 11428025] (i) Knapp S, Myers DS. J Org Chem 2002;67:2995. [PubMed: 11975558] (j) Zhu XM, Pachamuthu K, Schmidt RR. Org Lett 2004;6:1083. [PubMed: 15040728] (k) Zhu XM, Schmidt RR. Chem Eur J 2004;10:875. (l) Thayer DA, Yu HN, Galan MC, Wong CH. Angew Chem Int Ed 2005;44:4596. (m) Bertozzi CR, Kiessling LL. Science 2001;291:2357. [PubMed: 11269316] (n) Galonic DP, Ide ND, van der Donk W, Gin DY. J Am Chem Soc 2005;127:7359. [PubMed: 15898784]

10. Roy, R. Carbohydrate Chemistry. Boons, G-J., editor. Blackie; London: 1998. p. 243Lee, YT.; Lee, RC., editors. Neoglycoconjugates: Preparation and Applications. Academic; San Diego: 1994. (c) Ichikawa Y, Matsukawa Y, Isobe M. J Am Chem Soc 2006;128:3934. [PubMed: 16551100] (d) Ladmiral V, Mantovani G, Clarkson GJ, Cauet S, Irwin JL, Haddleton DM. J Am Chem Soc 2006;128:4823. [PubMed: 16594719] (e) Hotha S, Kashyap S. J Org Chem 2006;71:364. [PubMed: 16388660]

11. Dawson PE, Kent SBH. Ann Rev Biochem 2000;69:923. [PubMed: 10966479]

12. (a) Dwek RA, Butters TD. Chem Rev 2002;102:283. (b) Varki A. Glycobiology 1993;3:97. [PubMed: 8490246]Lehmann, J. Carbohydrates: Structure and Biology. Thieme; Stuttgart: 1998.

13. (a) He Y, Hinklin RJ, Chang J, Kiessling L. Org Lett 2004;6:4479. [PubMed: 15548055] (b) Grandjean C, Boutonnier A, Guerreiro C, Fournier JM, Mulard LA. J Org Chem 2005;70:7123. [PubMed: 16122231] (c) Wen S, Guo Z. Org Lett 2001;3:3773. [PubMed: 11700135] (d) Mizuno M, Goto K, Miura T. Chem Lett 2005;34:426. (e) Kaneshiro CM, Michael K. Angew Chem Int Ed 2006;45:1077. (f) Mandal M, Dudkin VY, Geng X, Danishefsky SJ. Angew Chem Int Ed 2004;43:2557. (g) Lin H, Walsh CT. J Am Chem Soc 2004;126:13998. [PubMed: 15506762] (h) Tanaka H, Iwata Y, Takahashi D, Adachi M, Takahashi T. J Am Chem Soc 2005;127:1630. [PubMed: 15700984] (i) Doores KJ, Mimura Y, Dwek RA, Rudd PM, Elliott T, Davis BG. Chem Commun 2006:1401. (j) Liu H, Wang L, Brock A, Wong CH, Schulz PG. J Am Chem Soc 2003;125:1702. [PubMed: 12580587] (k) Brik A, Yang YY, Ficht S, Wong CH. J Am Chem Soc 2006;128:5626. [PubMed: 16637616] (1) Hang HC, Bertozzi CR. Acc Chem Res 2001;34:727. [PubMed: 11560472]

14. Nyong AM, Rainier JD. J Org Chem 2005;70:746. [PubMed: 15651836]

15. (a) Salim M, Capretta A. Tetrahedron 2000;56:8063. (b) Gibe R, Kerr MA. J Org Chem 2002;67:6247. [PubMed: 12182672]

16. It can be similarly inferred that the reaction of rhodium carbenoids with protein-based tryptophan described by Francis is likely not compatible with the presence of nucleophilic sulfur as in cysteine and methionine.

17. Creary X. J Am Chem Soc 1980;102:1502.

18. Scott LT, Sumpter CA. Org Synth 1990;69:180.

19. Holton TL, Shechter H. J Org Chem 1995;60:4725. 
20. (a) House HO, Blankley CJ. J Org Chem 1968;33:53. (b) Ouihia A, Rene L, Badet B. Tetrahedron Lett 1992;33:5509. 


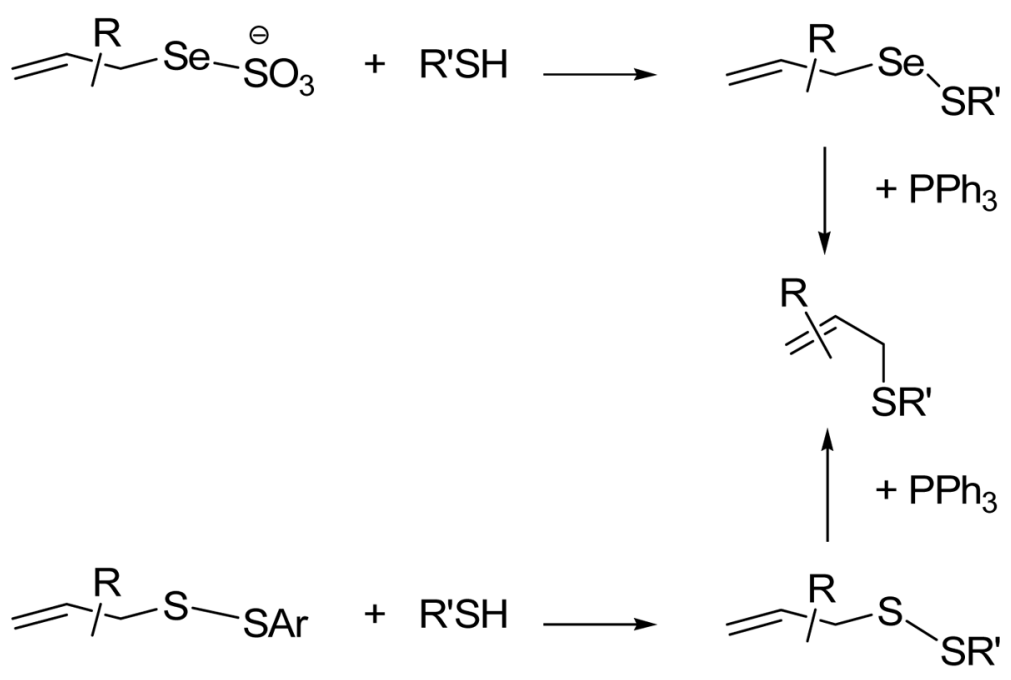

\section{$\mathrm{Ar}=$ 2-pyridyl, 5-nitro-2-pyridyl, 2-benzothiazolyl}

Scheme 1.

Dechalcogenative Allylic Selenosulfide and Disulfide Ligations 


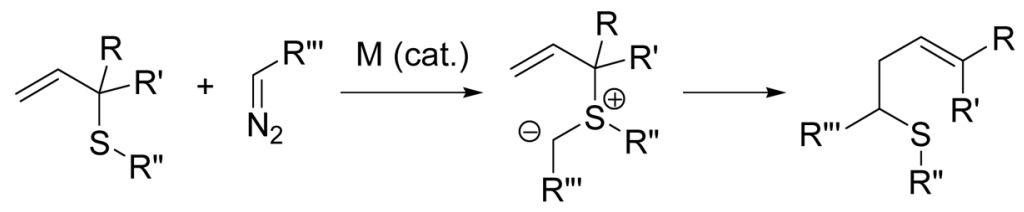

Scheme 2.

Reaction of metal carbenoids with allyl thio ethers 


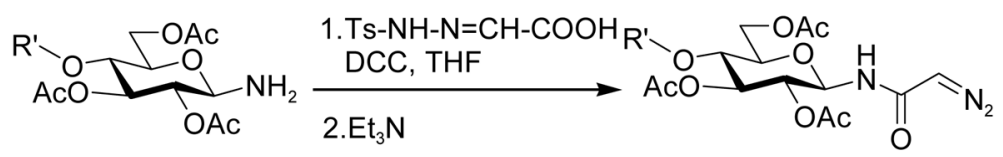

13: $R^{\prime}=$ Ac $(46 \%)$

14: $R^{\prime}=$ tetraacetyl- $\beta$-D-glucosyl $(59 \%)$

Scheme 3.

Preparation of diazo amides $\mathbf{1 3}$ and $\mathbf{1 4}$ 
Table 1

Reaction of carbenoids with cysteine derivatives.

2: $\mathrm{R}_{2=\mathrm{Me}}$


Table 2

Reaction of carbenoids with $S$-allyl glutathione.

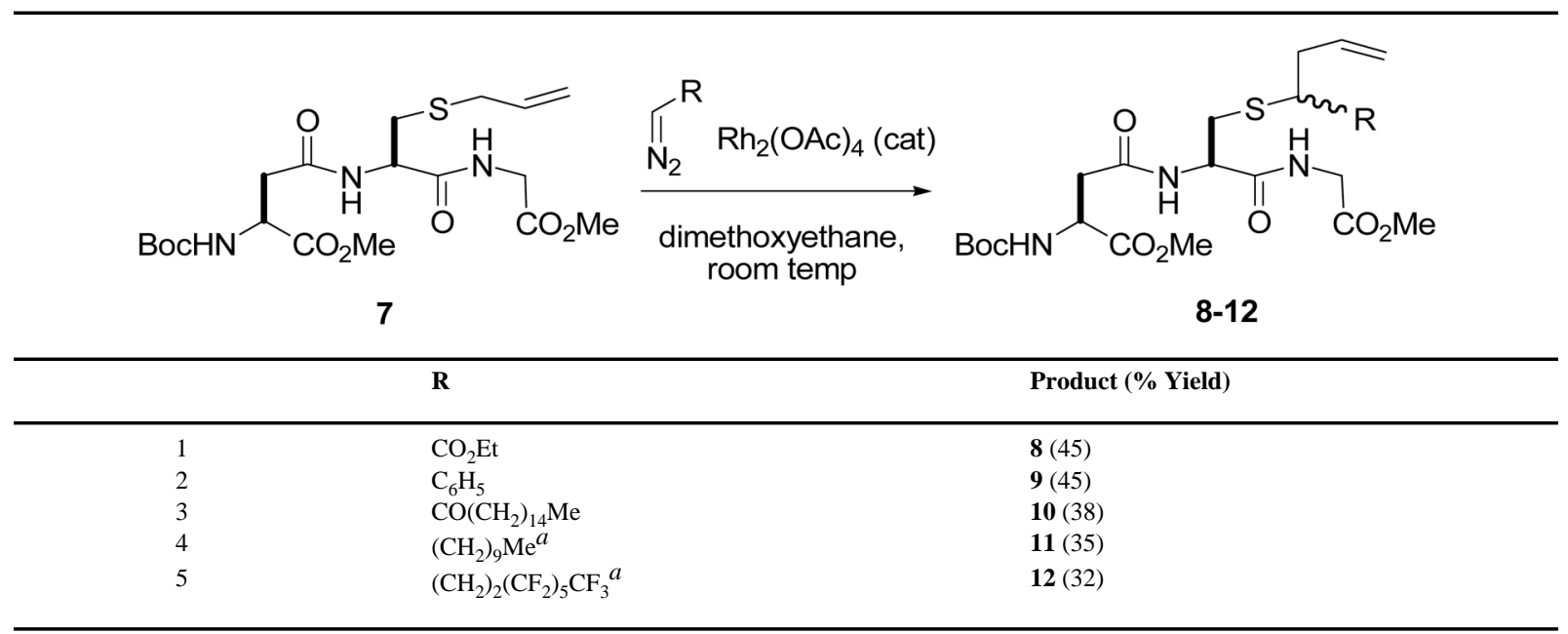

${ }^{a}$ Diazo precursors to $\mathbf{1 1}$ and $\mathbf{1 2}$ were prepared from the hydrazones with $\mathrm{Pb}(\mathrm{OAc}) 4$ and were used immediately. 
Table 3

$\mathrm{Rh}_{2}(\mathrm{OAc})_{4}$ Catalyzed Glycosylation

Substrate

